\title{
Long-Term Outcomes after Surgery for Appendiceal Mucinous Tumours
}

\author{
Audrius Dulskas ${ }^{a}$ Tomas Poskus $^{b}$ Eligijus Poskus ${ }^{b}$ Kestutis Strupas ${ }^{b}$ \\ ${ }^{a}$ Department of Abdominal and Oncological Surgery, National Cancer Institute, Vilnius, Lithuania; \\ ${ }^{b}$ Center of Abdominal Surgery, Vilnius University Hospital Santaros Clinics, Vilnius, Lithuania
}

\section{Keywords}

Appendectomy - Mucinous appendiceal neoplasm . Mucocele - Pseudomyxoma peritonei - Cytoreductive surgery · Hyperthermic intraperitoneal chemotherapy, HIPEC

\section{Summary}

Background: Appendiceal mucinous tumour (AMT) is a very uncommon disease. We aimed to evaluate preoperative and postoperative characteristics as well as longterm outcomes of patients with appendiceal mucinous adenomas and peritoneal pseudomyxoma (PMP). Methods: A consecutive cohort of patients diagnosed with AMT were included in the study. Tumour recurrence and the development of PMP was diagnosed based on the patients' complaints, physical examination, imaging studies, and biopsy results. The calculation of survival rates was performed using the Kaplan-Meier method. Results: Between January 2003 and February 2013, 25 patients had AMT. 15 patients underwent appendectomy only $(60 \%), 5$ patients $(20 \%)$ right hemicolectomy, 4 patients $(16 \%)$ cytoreductive surgery (CRS) \pm hyperthermic intraperitoneal chemotherapy (HIPEC), and 1 patient (4\%) appendectomy with caecal resection. Another 2 patients underwent CRS and HIPEC 4 days and 3 years, respectively, following initial appendectomy. In the histopathologic examination, adenoma was diagnosed in 12 patients (48\%); among these, low-grade PMP was found in 1 patient (4\%). Low-grade appendiceal mucinous adenocarcinoma (AMCa) was diagnosed in 11 patients (44\%); among these, low-grade PMP was found in seven cases $(28 \%)$ and high-grade AMCa was diagnosed in 2 patients (8\%). 2 of 8 PMP patients did not undergo CRS \pm HIPEC because of comorbidities. The 5 -year overall survival was $100 \%$ for the adenomas and $62 \%$ for PMP patients. Conclusion: AMT is a very rare entity with a con- siderably good outcome even in disseminated disease. CRS and HIPEC is a gold standard treatment for PMP with excellent prognosis. However, our results could still be improved in the future.

(c) 2017 S. Karger GmbH, Freiburg

\section{Introduction}

Karl Rokitansky was the first to recognize and describe an appendiceal mucocele (appendiceal mucinous tumour (AMT)) in 1842 [1]. Mucocele is a rare pathology of the appendix ranging between 0.2 and $0.7 \%$ of all appendectomies [2-4]. Classification and management of AMT is very problematic for both clinicians and pathologists. These tumours may disseminate throughout the peritoneal cavity in the form of gelatinous deposits, termed peritoneal pseudomyxoma (PMP). PMP is the worst complication of AMT, caused by spontaneous or iatrogenic perforation of the appendix, which comes along with a high rate of morbidity and mortality [5, 6]. Several investigators have proposed different classifications with similar terminology to describe lesions of variable biologic potential [7-10].

The purpose of this study was to evaluate preoperative and postoperative characteristics as well as long-term outcomes of patients with appendiceal mucinous adenomas and PMP.

\section{Patients and Methods}

During the study period from January 2003 to August 2013, patients with the diagnosis of AMT were identified from the Vilnius University Hospital Santaros Clinics electronic database using International Classification of Disease 10th revision (ICD-10) codes. At the first step, we searched the patient list for ICD-10 code K35. At the second step, we separated these patients as malignant and benign neoplasms of the appendix by using ICD-10 codes C18.1 and D12.1

\section{KARGER}

(c) 2018 S. Karger GmbH, Freiburg

Fax +497614520714 
Fig. 1. Treatment algorithm for appendiceal mucinous tumours. $\mathrm{AMCa}=$ Appendiceal mucinous adenocarcinoma; $L G$ = low-grade; $\mathrm{HG}=$ high-grade; $\mathrm{CRS}$ = cytoreductive surgery; HIPEC $=$ hyperthermic intraperitoneal chemotherapy.

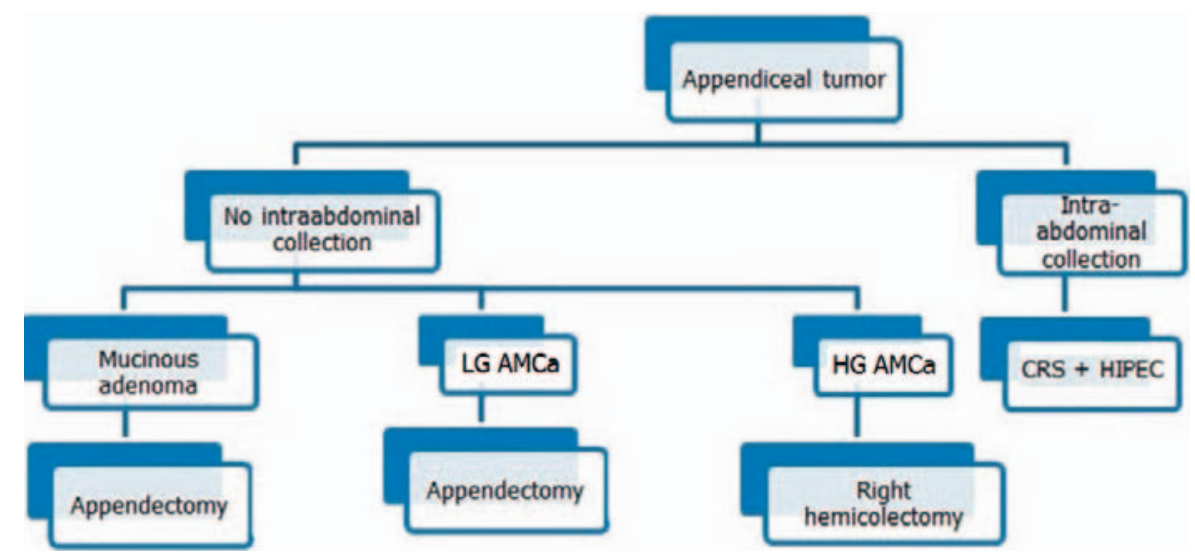

from the hospital electronic database system. Patients were evaluated regarding age, sex, symptom duration, major complaint, surgical procedure performed, pathologic diagnosis, and their follow-up outcomes.

In Lithuania mainly one classification system is used, i.e. that of the World Health Organization (WHO), which states that any neoplastic epithelial proliferation confined to the appendiceal mucosa is classified as an adenoma. AMT with extra-appendiceal neoplastic epithelium are classified as mucinous adenocarcinomas (AMCa) and subcategorized as low- or high-grade because increasingly severe cytoarchitectural atypia is associated with poorer outcome [8].

If PMP or AMCa is suspected, we perform whole-body computed tomography (CT) scan as well as abdominal and pelvic nuclear magnetic resonance imaging for staging.

For choosing the most appropriate procedure for different patients, we use our treatment algorithm (fig. 1): if the patient presents with AMCa and if there are fluid collections within the abdominal cavity (dissemination of mucin-producing neoplastic cells throughout the peritoneal cavity, either proven by frozen biopsy or seen ad oculi), patients should undergo cytoreductive surgery (CRS) with hyperthermic intraperitoneal chemotherapy (HIPEC), as carried out for PMP. When no intra-abdominal collection is evident, appendectomy with clear margins and abdominal exploration for possible PMP should be performed, possibly taking peritoneal biopsies in suspicious cases for PMP. If margins are positive and high-grade AMCa is diagnosed, right hemicolectomy should be added.

Details of CRS and HIPEC have been described elsewhere [11]. In brief, resections were done as needed to achieve complete cytoreduction, including excision of previous scar and port sites, anterior abdominal wall peritonectomy, splenectomy, cholecystectomy, greater and lesser omentectomy, diaphragmatic and pelvic peritonectomies, stripping of peritoneum over omental bursa and porta hepatis, and visceral peritonectomies. HIPEC was performed using a closed technique for $90 \mathrm{~min}$ before performing any anastomoses. Mitomycin C was used with a dose of $40 \mathrm{mg}$; i.e., $30 \mathrm{mg}$ given at time zero and $10 \mathrm{mg}$ given 30 min later. The target outflow temperature was maintained at $41-42^{\circ} \mathrm{C}$, which requires an inflow temperature of $42-43^{\circ} \mathrm{C}$. The patients were transferred to the intensive care unit of the surgical ward during the first $72 \mathrm{~h}$ of the postoperative period. No systemic chemotherapy was later prescribed.

Clinical follow-up with abdominal CT and tumour markers was done every 3 months for the first 2 years and every 6 months thereafter. Tumour recurrence was diagnosed based on the patients' complaints, physical examination, imaging studies, and biopsy results.

\section{Statistical Analysis}

Continuous variables are expressed as median (range). Overall survival (OS) was calculated from the date of CRS/HIPEC treatment to the date of death. Estimates of survival were calculated using the Kaplan-Meier method. Differences were calculated with a log-rank test. A total of 25 patients were entered into the registry. The data collected was analysed using SPSS software (Statistical Package for the Social Sciences; SPSS Inc., Chicago, IL, USA). A p-value of $<0.05$ was considered statistically significant.

\section{Results}

Between January 2003 and August 2013, a total of 3,100 patients with the preoperative diagnosis of appendicitis were treated in our centre. $25(0.8 \%)$ of these patients had AMT. The average age of the patients was 68.4 years (range: $41-90$ years). 12 (48\%) of the 25 patients were female, and 13 (52\%) were male. The body mass index was $22.5 \pm 3.4 \mathrm{~kg} / \mathrm{m}^{2}$. The duration of the symptoms was variable, ranging between $12 \mathrm{~h}$ and 10 years, with a median of 6.8 months. Most of the patients (80\%) complained of abdominal pain, while less patients had palpable abdominal mass (12\%), vomiting (8\%), and weight loss (8\%). There was no abnormality in biochemistry or complete blood count of the patients except for leucocytosis in 12 patients (48\%).

All patients were operated. The surgical procedures performed are reported in table 1 . In our series, 15 patients underwent appendectomy; 2 of them had CRS and HIPEC after 4 days and 3 years, respectively, due to PMP. 5 patients underwent right hemicolectomy: 3 of them due to infiltrate in the right iliac fossa, 1 with histology of high-grade mucinous adenocarcinoma and positive nodal disease spread, and 1 had synchronous ascending colon cancer. Caecal resection had been performed in one of the patients to obtain negative surgical margins, since the appendiceal wall was contiguous with the caecum and intraoperative pathology indicated benign mucocele. 4 patients underwent CRS \pm HIPEC (depending on physical state) because of PMP found preoperatively.

Histopathological findings according to the classification of the WHO, which is used in Lithuania, are shown in figure 2. Mucinous adenoma was diagnosed in 12 patients (48\%), among them one with PMP; low-grade mucinous adenocarcinoma in 11 patients (44\%), among them 6 (28\%) patients with PMP and 1 patient in which PMP developed 3 years later; and high-grade mucinous adenocarcinoma in $2(8 \%)$ patients.

Postoperative complications of 8 patients (32\%) are as follows (details can be found in table 1): anastomotic leak after CRS and HIPEC: 1 (4\%), wound infection: $1(4 \%)$, pulmonary embolism (PE): 1 (4\%), cerebrovascular accident: 1 (4\%), bowel obstruction: $1(4 \%)$, cellulitis: $1(4 \%)$, atrial fibrillation: $1(4 \%)$, renal failure: 1 (4\%), and wound dehiscence: 1 (4\%). One of these patients was reoperated and underwent repeated surgery 5 days after CRS + 
Table 1. Operative findings and surgical procedures performed in 25 patients with appendiceal mucinous tumours

\begin{tabular}{llll}
\hline Operative findings & Surgical procedure & $\mathrm{n}(\%)$ & Complications (CD) \\
\hline $\begin{array}{l}\text { Enlarged/ } \\
\text { changed appendix }\end{array}$ & $\begin{array}{l}\text { appendectomy } \\
\text { only }\end{array}$ & $15(60)$ & $\begin{array}{l}\text { wound infection 1 (4\%) (CD I) } \\
\text { leg cellulitis 1 (4\%) (CD II) } \\
\text { deep vein thrombosis 1 (4\%) (CD II) } \\
\text { bowel obstruction 1 (4\%) (CD II) } \\
\text { cerebrovascular accident 1 (4\%) (CD V) }\end{array}$ \\
$\begin{array}{c}\text { Infiltrate in the right } \\
\text { iliac fossa or positive } \\
\text { nodal disease }\end{array}$ & $\begin{array}{l}\text { right } \\
\text { hemicolectomy }\end{array}$ & $5(20)$ & $\begin{array}{l}\text { PE 1 (4\%) (CD V) } \\
\text { wound dehiscence 1 (4\%) (CD I) }\end{array}$ \\
$\begin{array}{c}\text { Intraperitoneal } \\
\text { collections }\end{array}$ & CRS \pm HIPEC & $4(16)$ & anastomotic leak 1 (4\%) (CD IVa) \\
$\begin{array}{c}\text { Infiltrative base of } \\
\text { appendix }\end{array}$ & $\begin{array}{l}\text { appendectomy + } \\
\text { caecal resection }\end{array}$ & $1(4)$ & - \\
\hline
\end{tabular}

$\mathrm{PE}=$ Pulmonary embolism; $\mathrm{CRS}=$ cytoreductive surgery; $\mathrm{HIPEC}=$ hyperthermic intraperitoneal chemotherapy; $\mathrm{CD}=$ Clavien-Dindo.
HIPEC for anastomotic leak; Hartman's procedure was performed. There were two postoperative deaths (8\%): a 70 -year-old male with synchronous ascending colonic cancer died 6 days after right hemicolectomy due to $\mathrm{PE}$, and a 78-year-old male died 12 days after appendectomy due to a cerebrovascular accident. The average postoperative length of hospital stay was 14 (3-46) days.

The average follow-up period was 27.2 (12-61) months. 5 patients were lost during follow-up - all of them had mucinous adenoma. 2 patients died of disease-unrelated causes. 1 patient developed PMP in the course of follow-up within 3 years and underwent CRS + HIPEC. No other recurrences were seen. The 5-year OS was $62 \%$, as calculated by the Kaplan-Meier product limit estimate for PMP patients. Due to the advanced age and chronicity of their disease, the precise cause of death of some patients could not be adequately determined in this analysis.

\section{Discussion}

We found that the 5-year OS of patients with PMP was $62 \%$.

During the past several decades, various controversies in the field of AMT have been debated in the literature; these were mainly related to treatment options.

As in a study by Smeenk et al. [12], we also had one case of PMP in the mucinous adenoma group. We assume that there were no signs of invasive carcinoma in the appendix, but PMP still developed later either because the primary specimen was not examined thoroughly or because there was contamination of tumour cells during the operation. Misclassification cannot be excluded as well.

There is little consensus on the optimal choice of procedure (right hemicolectomy versus appendectomy) as well as the approach (laparoscopic versus laparotomy) for the treatment of AMT. Laparoscopic appendectomy for benign mucinous tumour removal has been described (traditional and single incision [13, 14]), but caution has also been suggested because of the risk of tumour seeding. It is probably safer to convert to an open procedure if AMT is visualized during a laparoscopic approach $[2,3,13,14]$. All except

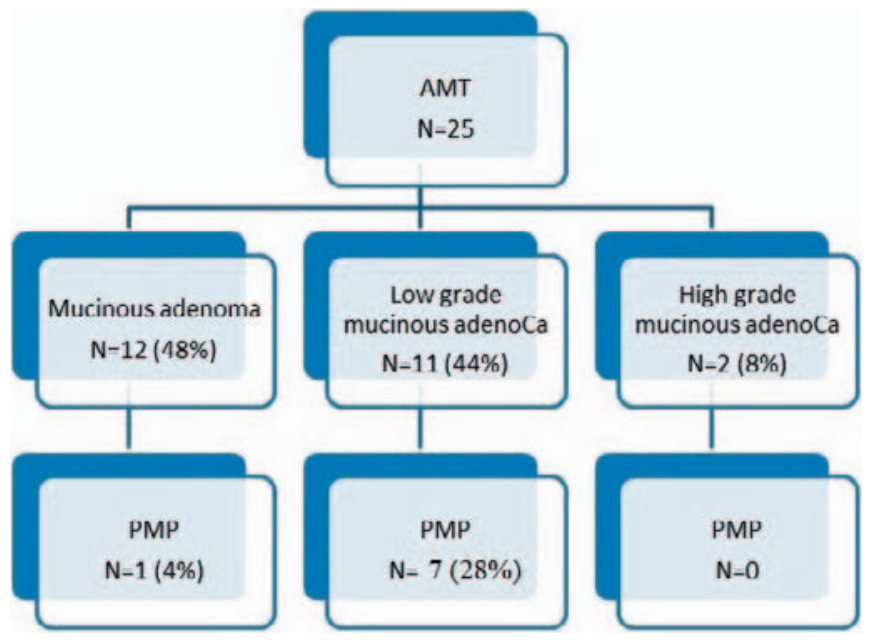

Fig. 2. Histopathological findings according to the WHO classification for appendiceal mucinous tumours (AMT) and risk for peritoneal pseudomyxoma (PMP).

one of the operations performed in our series were open. The peritoneal surfaces surrounding the appendix, liver, left paracolic gutter, omentum, and pelvis must be inspected for tumorous deposits or mucus. If detected, a sample should be sent for histopathologic or cytological examination. Generally, AMCa spread through the wall of the appendix into the intraperitoneal cavity and rarely metastasize to the lymph nodes $[15,16]$. On this account, a less aggressive surgical approach than right colectomy has been suggested; thus, tumours confined to the appendix without evidence of peritoneal dissemination or PMP (adenoma or low-grade AMCa) are resected $[3,4,10,17]$. We disagree with sending frozen sections for histology - the report is often very inaccurate, lacking information on the progression of the disease, and the specimen is spoilt for better elective examination. Turaga et al. [15] reported of 2,100 patients with appendiceal adenocarcinoma. They concluded that there was no therapeutic benefit of a right hemicolectomy in the setting of known node-positive or metastatic disease. Right hemicolectomy should be performed in the setting where the tumour cannot be 
cleared otherwise or when the need for staging is strong enough to warrant the morbidity of the operation [15]. Same results were shown by Gonzalez-Moreno and Sugarbaker [18] who reviewed 501 patients diagnosed with appendiceal epithelial neoplasm.

Now most authors agree that complete cytoreduction with HIPEC rather than debulking surgery is the gold standard for PMP treatment $[13,17,19-21]$. The main goal of PMP treatment is the prevention of locoregional recurrence rather than systemic disease. Wagner et al. [22] retrospectively analysed 282 patients undergoing CRS and HIPEC. They concluded that the extent of CRS does not affect the morbidity but prolongs the survival [22]. Recently a large multicentre retrospective study by Chua et al. [23] with more than 2,000 patients with PMP was published. The authors concluded that the 10-year OS rate after CRS and HIPEC was more than $63 \%$. Moran et al. [24] have just recently shown a 5-year OS rate of $84 \%$ in patients with appendiceal tumours and PMP who had complete cytoreduction. In our cohort, the 5-year OS for patients with PMP was only $62 \%$. This is worse than stated in the large studies mentioned above. There might be several reasons for a worse outcome. First of all, the completeness of CRS might be doubtful since we are only achieving our learning curve. Secondly, not all of the patients adhere to the strict follow-up recommendations and may come back with worsened disease. Lastly, we cannot obtain the exact causes of death from a single registry as we do not have such a registry in Lithuania. Thus, it might be possible that the cause of death was not due to the progression of the disease. Some authors argue for the need of CRS and HIPEC, stating that serial debulking surgeries have the same efficiency and are associated with less morbidity $[25,26]$. Some authors advocate for watchful waiting of PMP if there is low grade and low spread of the disease [27]. Others advocate early risk-reducing laparoscopic CRS for some tumours [28]. Both of these studies have a short-term follow-up and therefore should be very cautiously recommended as treatments.
We had eight PMP cases (seven low-grade and one mucinous adenoma). 4 patients underwent CRS and HIPEC at our hospital (the procedure was first started in Lithuania at our hospital in April 2011). 1 patient with low-grade PMP and severe comorbidities underwent appendectomy and removal of mucinous masses only. He was disease-free for 60 months (later he was lost during follow-up). A second patient with low-grade PMP underwent right hemicolectomy and removal of mucinous masses. All patients with $\mathrm{AMCa}$ and PMP are followed up concerning the progression of disease for 26 months (3-61 months) on average. Current evidence demonstrates median OS rates of 16.3 years, with a 15 -year survival rate of $59 \%$ [23].

The main weakness of our study is the retrospective nature of inclusion into the database and the small size of the cohort.

We are of the opinion that all patients who have had mucinous deposits or some other signs of perforation during surgery as well as those patients microscopically showing mucus infiltration to the appendiceal serosa require at least 5 years of surveillance. In our series, 1 patient developed PMP 3 years after appendectomy performed at another hospital.

\section{Conclusions}

AMT is a very rare entity with considerably good outcome even in disseminated disease. CRS and HIPEC is a gold standard treatment for PMP with excellent prognosis. However, our results could still be improved in the future.

\section{Disclosure Statement}

The authors declare that there is no conflict of interest.

\section{References}

1 Rokitansky CF: A Manual of Pathological Anatomy. Vol. 2. Philadelphia, PA, Blanchard \& Lea, 1855

2 Honnef I, Moschopulos M, Roeren T: Appendiceal mucinous cystadenoma. Radiographics 2008;28:15241527.

3 Karakaya K, Barut F, Emre AU, et al: Appendiceal mucocele: case reports and review of current literature. World J Gastroenterol 2008;14:2280-2283.

4 de Abreu Filho JG, de Lira EF: Mucocele of the appendix - appendectomy or colectomy? Rev Bras Coloproct 2011;31:276-284.

5 Stocchi L, Wolff BG, Larson DR, et al: Surgical treatment of appendiceal mucocele. Arch Surg 2003;138: 585-590.

6 Dixit A, Robertson JHP, Mudan SS, et al: Appendiceal mucocoeles and pseudomyxoma peritonei. World J Gastroenterol 2007;13:2381-2384.

7 Panarelli NC, Yantiss RK: Mucinous neoplasms of the appendix and peritoneum. Arch Pathol Lab Med 2011; 135:1261-1268.

8 Carr NJ, Sobin LH: Adenocarcinoma of the appendix. WHO classification of tumors of the digestive system. Lyon, IARC, 2010, pp 93-98.
9 American Joint Committee on Cancer 2012. Appendix. https://cancerstaging.org/Pages/default.aspx.

10 Pai RK, Longacre TA: Appendiceal mucinous tumors and pseudomyxoma peritonei: histologic features, diagnostic problems, and proposed classification. Adv Anat Pathol 2005;12:291-311.

11 El Halabi H, Gushchin V, Francis J, et al: The role of cytoreductive surgery and heated intraperitoneal chemotherapy (CRS/HIPEC) in patients with highgrade appendiceal carcinoma and extensive peritoneal carcinomatosis. Ann Surg Oncol 2012;19:110-114.

12 Smeenk RM, van Velthuysen MLF, Verwaal VJ, et al: Appendiceal neoplasms and pseudomyxoma peritonei: a population based study. Eur J Surg Oncol 2008;34: 196-201.

13 Yoshida Y, Sato K, Tada T, et al: Two cases of mucinous cystadenoma of the appendix successfully treated by laparoscopy. Case Rep Gastroenterol 2013;7:44-48.

14 Park KB, Park JS, Choi GS, et al: Single-incision laparoscopic surgery for appendiceal mucoceles: safety and feasibility in a series of 16 consecutive cases. J Korean Soc Coloproctol 2011;27:287-292.
15 Turaga KK, Pappas S, Gamblin TC: Right hemicolectomy for mucinous adenocarcinoma of the appendix: just right or too much? Ann Surg Oncol 2013;20:10631067

16 Fuchs CK, Kuruvilla YCK, Angst E, et al: Appendiceal mucocele in an elderly patient: how much surgery? Case Rep Gastroenterol 2011;5:516-522.

17 McDonald JR, O'Dwyer ST, Rout S, et al: Classification of and cytoreductive surgery for low-grade appendiceal mucinous neoplasms. Br J Surg 2012;99:987-992.

18 Gonzalez-Moreno S, Sugarbaker PH: Right hemicolectomy does not confer a survival advantage in patients with mucinous carcinoma of the appendix and peritoneal seeding. Br J Surg 2004;91:304-311.

19 Andreasson H, Graf W, Nygren P, et al: Outcome differences between debulking surgery and cytoreductive surgery in patients with pseudomyxoma peritonei. Eur J Surg Oncol 2012;38:962-968.

20 Sugarbaker PH: New standard of care for appendiceal epithelial neoplasms and pseudomyxoma peritonei syndrome? Lancet Oncol 2006;7:69-76. 
21 Carr NJ, Finch J, Ilesley IC, et al: Pathology and prognosis in pseudomyxoma peritonei: a review of 274 cases. J Clin Pathol 2012;65:919-923.

22 Wagner PL, Austin F, Maduekwe U, et al: Extensive cytoreductive surgery for appendiceal carcinomatosis: morbidity, mortality, and survival. Ann Surg Oncol 2013;20:1056-1062.

23 Chua TC, Moran BJ, Sugarbaker PH et al: Early- and long-term outcome data of patients with pseudomyxoma peritonei from appendiceal origin treated by a strategy of cytoreductive surgery and hyperthermic intraperitoneal chemotherapy. J Clin Oncol 2012;30 2449-2456.
24 Moran B, Cecil T, Chandrakumaran K, Arnold S, Mohamed F, Venkatasubramaniam A: The results of cytoreductive surgery and hyperthermic intraperitoneal chemotherapy in 1200 patients with peritoneal malignancy. Colorectal Dis 2015;17:772-778.

25 Dayal S, Taflampas P, Riss S, et al: Complete cytoreduction for pseudomyxoma peritonei is optimal but maximal tumor debulking may be beneficial in patients in whom complete tumor removal cannot be achieved. Dis Colon Rectum 2013;56:1366-1372.

26 Miner TJ, Shia J, Jaques DP, et al: Long-term survival following treatment of pseudomyxoma peritonei. Ann Surg 2005;241:300-308.
27 Zih FSW, Wong-Chong N, Hummel C et al: Mucinous tumor of the appendix with limited peritoneal spread: is there a role for expectant observation? Ann Surg Oncol 2014;21:225-231.

28 Fish R, Selvasekar C, Crichton P, et al: Risk-reducing laparoscopic cytoreductive surgery and hyperthermic intraperitoneal chemotherapy for low-grade appendiceal mucinous neoplasm: early outcomes and technique. Surg Endosc 2014;28:341-345. 